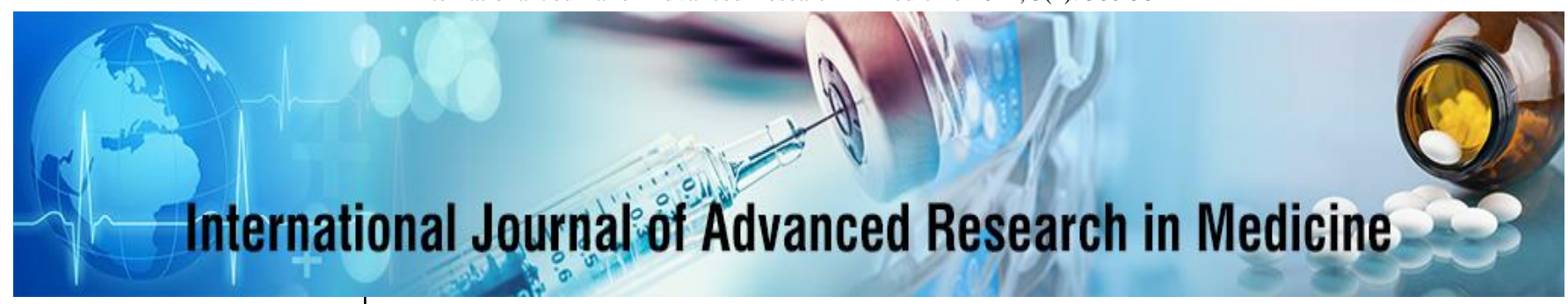

E-ISSN: 2706-9575

P-ISSN: 2706-9567

IJARM 2021; 3(2): 580-582

Received: 19-08-2021

Accepted: 21-10-2021

Mustafa Alaziz

MBBS, MS, Uwaydah Clinic,

Family Practice, Houston,

Texas, United States of

America

Yusra Al Saffar BS

Virginia Commonwealth

University, Virginia,

United States of America

\section{Pregnancy in bicornuate uterus: A case report and literature review}

\author{
Mustafa Alaziz and Yusra Al Saffar BS
}

DOI: $\underline{\text { https://doi.org/10.22271/27069567.2021.v3.i2i.308 }}$

\begin{abstract}
Introduction: The bicornuate uterus is a rare congenital anomaly associated with an increased risk of obstetric complications such as recurrent miscarriage, preterm labor, and malpresentation; however, a successful pregnancy is attainable, but it needs close prenatal monitoring; in some cases, surgical intervention is required.

Case Presentation: 44-year-old female, G10P5A4, seven weeks gestation, presented for prenatal care. She is a known case of the bicornuate uterus; she had a history of left tubal ligation, three dilatation and curettage, in addition to three C-Section. Her most recent ultrasound showed a bicornuate uterus with an intrauterine sac at the right horn and left adnexal mass.

Discussion: Most cases of the bicornuate uterus are asymptomatic, diagnosed during routine evaluation; however, some cases presented with dysmenorrhea and menorrhagia. A bicornuate uterus can be isolated or associated with other anomalies such as renal agenesis and vaginal septum.

Conclusion: Ultrasound is the initial diagnostic test to detect bicornuate uterus, and magnetic resonance imaging represents the gold standard diagnostic imaging study. The detection of an asymptomatic bicornuate uterus requires rigorous prenatal monitoring; however, cases of bicornuate uterus with a history of obstetric complications necessitate surgical intervention with metroplasty or laparoscopy.
\end{abstract}

Keywords: bicornuate uterus, pregnancy, miscarriage

\section{Introduction}

Congenital uterine anomalies result from abnormal development of Müllerian ducts during fetal life ${ }^{[1]}$. However, their effect on reproductive outcomes is unclear ${ }^{[2]}$. Uterine congenital anomalies ranged between two extremes extended from the mild form such as the arcuate uterus, which has a minimal clinical significance, to a more severe form such as Müllerian agenesis. All other subtypes of congenital uterine anomalies ranged between these two extremes: including unicornuate or bicornuate uteri, uterus didelphys, and septate uterus ${ }^{[3]}$.

A bicornuate uterus is a class IV of Mullerian anomalies ${ }^{[4]}$, and it can be complete or partial, resulting from an abnormal fusion of Mullerian ducts ${ }^{[5]}$. The bicornuate uterus is a rare anomaly and represents about $0.4 \%$ of the general population ${ }^{[6]}$. A bicornuate uterus is associated with obstetric complications such as recurrent pregnancy loss, preterm labor, and malpresentation ${ }^{[3,7]}$. Despite the increased risk of complications associated with a bicornuate uterus, a successful pregnancy can be achieved, but it requires aggressive prenatal monitoring or surgical unification depending on an individual basis.

We present a case of a 44-year-old female who was diagnosed with a bicornuate uterus, and she had multiple successful pregnancies and recurrent pregnancy loss.

\section{Case Presentation}

A 44-year-old female present to our primary care clinic with 7 weeks gestation for prenatal care. The patient is G10 P5A4. The patient was diagnosed with a bicornuate uterus and underwent left fallopian tube ligation in 2009. This is her tenth pregnancy; she had five successful pregnancies and four pregnancy losses. She had a history of three dilation and curettage $(\mathrm{D} \& \mathrm{C})$ and three $\mathrm{C}$-sections. Her quantitative HCG level on December 10 was 5193 MIU/ML. On December 21, the quantitative HCG level was 5872 MIU/ML. All other Lab results were within normal limits.
Corresponding Author: Mustafa Alaziz MBBS, MS, Uwaydah Clinic, Family Practice, Houston,

Texas, United States of America 
The most recent transabdominal/transvaginal ultrasound of her current pregnancy showed a bicornuate uterus; the right uterus measured $11.0 \times 4.0 \times 4.3 \mathrm{~cm}$, and left uterus measured $9.0 \times 4.5 \times 4.0 \mathrm{~cm}$. an intrauterine sac-like structure measured $1.2 \mathrm{~cm}$ is identified at the right horn of the bicornuate uterus without a fetal pole or cardiac activity corresponding to an estimated gestational age five weeks and six days, which may represent an intrauterine pregnancy, blighted ovum or less likely pseudo gestational sac secondary to ectopic pregnancy. A complex structure measuring $5.6 \times 3.8 \times 3.1 \mathrm{~cm}$ was noted in the left adnexa, which could represent a large ovarian cyst, but a cystic mass or ectopic pregnancy is not excluded. There was no evidence of subchorionic hemorrhage.

The pregnancy was determined to be non-viable because the HCG level was not doubled every 72 hours, and the ultrasound did not show any cardiac activity. Misoprostol was given to the patient to expel the non-viable embryo and to terminate the pregnancy.

\section{Discussion}

Most cases of the bicornuate uterus are asymptomatic in their adolescence ${ }^{[8]}$. Some cases may present with menorrhagia or dysmenorrhea, while others are diagnosed during routine evaluation for pregnancy; in addition, many cases are diagnosed when they present with obstetric complications. The physical exam is usually insignificant unless other abnormalities are present, such as genitourinary anomalies.

In some cases, a vaginal septum can be associated with a bicornuate uterus, which may result in dyspareunia, or the inability to control menstrual bleeding with tampon in such cases. Removal of the vaginal septum exposed the double cervix and uterus ${ }^{[9]}$.

Congenital renal defects can be seen with Mullerian anomalies due to the association between the development of the Mullerian duct, mesonephric duct, and urogenital sinus. Renal agenesis is the most common anomalies and is usually associated with the didelphys uterus. However, it can be seen with the bicornuate uterus ${ }^{[10]}$.

In a few cases of bicornuate uterus, there is an increased risk of rupture uterus if the zygote Implanted in the rudimentary horn of the bicornuate uterus because it is unable to expand like a normal uterus does to accommodate a growing fetus [11].

The diagnosis of the bicornuate uterus is primarily based on imaging which also directs the treatment. Ultrasound is the initial imaging technique to detect bicornuate uterus; it is cost-effective and widely available. 3D ultrasound has higher sensitivity and specificity than 2D ultrasound to differentiate bicornuate uterus from septate uterus ${ }^{[12]}$. Hysterosalpingography is another imaging modality that helps in the assessment of infertility ${ }^{[13]}$. Magnetic resonance imaging is the typical imaging modality to diagnose bicornuate uterus, and it is usually used for indeterminate cases ${ }^{[13]}$.

The treatment of the bicornuate uterus depends on patient presentation. In asymptomatic cases that are diagnosed on routine evaluation during pregnancy, then it needs close monitoring to prevent obstetric complications ${ }^{[9]}$. In cases presented with complications, such as recurrent miscarriage, recurrent preterm labor, then it requires surgical intervention, which is either by Strassman metroplasty or laparoscopy ${ }^{[14,15]}$. The Laparoscopic approach is superior to metroplasty as it is associated with fewer operative and post-operative complications ${ }^{[15]}$.

\section{Conclusion}

A bicornuate uterus can be an isolated finding, or it can be associated with other congenital anomalies. It can be asymptomatic, or it can be present with obstetric complications. The detection of the bicornuate uterus in a patient without a significant history does not mandate intervention; however, it requires close prenatal monitoring and management. For patients with a bicornuate uterus and a history of recurrent abortion or preterm labor, a unification procedure should be considered.

\section{References}

1. Moore KL, Persaud TVN, Torchia MG. The Urogenital System. In Before We Are Born: Essentials of Embryology and Birth Defects. ( $7^{\text {th }}$ edn). Saunders/Elsevier: Philadelphia, PA, 2008, 162-189.

2. Caserta D, Mallozzi M, Meldolesi C, et al. Pregnancy in a unicornuate uterus: A case report. J Med Case Rep. 2014;8:130.

3. Chan YY, Jayaprakasan K, Tan A, Thornton JG, Coomarasamy A, Raine-Fenning NJ. Reproductive outcomes in women with congenital uterine anomalies: A systematic review. Ultrasound Obstet Gynecol. 2011;38:371-382.

4. The American Fertility Society classifications of adnexal adhesions, distal tubal occlusion, tubal occlusion secondary to tubal ligation, tubal pregnancies, müllerian anomalies and intrauterine adhesions. Fertil Steril. 1988;49(6):944-55.

5. Roly ZY, Backhouse B, Cutting A, Tan TY, Sinclair $\mathrm{AH}$, Ayers KL et al. The cell biology and molecular genetics of Müllerian duct development. Wiley Interdiscip Rev Dev Biol. 2018;7(3):e310.

6. Chan YY, Jayaprakasan K, Zamora J, Thornton JG, Raine-Fenning N, Coomarasamy A. The prevalence of congenital uterine anomalies in unselected and highrisk populations: a systematic review. Hum Reprod Update. 2011;17(6):761-71.

7. Venetis CA, Papadopoulos SP, Campo R, Gordts S, Tarlatzis BC, Grimbizis GF. Clinical implications of congenital uterine anomalies: A meta-analysis of comparative studies. Reprod Biomed Online. 2014;29(6):665-83.

8. Itana de Mattos Pinto E Passos, Renata Lopes Britto. Diagnosis and treatment of müllerian malformations. Taiwan J Obstet Gynecol. 2020;59(2):183-188.

9. Letterie GS. Management of congenital uterine abnormalities. Reprod Biomed Online. 2011;23(1):4052.

10. Hall-Craggs MA, Kirkham A, Creighton SM. Renal and urological abnormalities occurring with Mullerian anomalies. J Pediatr Urol. 2013;9(1):27-32.

11. Kore S, Pandole A, Akolekar R, et al. Rupture of left horn of bicornuate uterus at twenty weeks of gestation. J Postgrad Med. 2000;46(1):39-40.

12. Deutch TD, Abuhamad AZ. The role of 3-dimensional ultrasonography and magnetic resonance imaging in the diagnosis of müllerian duct anomalies: a review of the literature. J Ultrasound Med. 2008;27(3):413-23. 
13. Behr SC, Courtier JL, Qayyum A. Imaging of müllerian duct anomalier. Radiographics. 2012;32(6):E233-50.

14. Strassmann EO. Fertility and unification of double uterus. Fertil Steril. 1966;17(2):165-76.

15. Alborzi S, Asefjah H, Amini M, Vafaei H, Madadi G, Chubak $\mathrm{N}$ et al. Laparoscopic metroplasty in bicornuate and didelphic uteri: feasibility and outcome. Arch Gynecol Obstet. 2015;291(5):1167-71. 